\title{
Comparing the efficacy of desmopressin and oxybutynin combination therapy and desmopressin monotherapy in children with primary nocturnal enuresis; a randomized clinical trial
}

\author{
Yalda Ravanshad', Anoush Azarfar ${ }^{2}$, Afshin Ghalegolab-Behbahan ${ }^{3}$, Fakhrosadat Mortazavi ${ }^{3}$, Sonia \\ Ahmadzadeh $^{4}$, Fereshteh Ghorat ${ }^{5}$, Samira Foji ${ }^{6}$, Elham Iziy ${ }^{5,7}$, Marjan Vejdani ${ }^{8^{*}}$

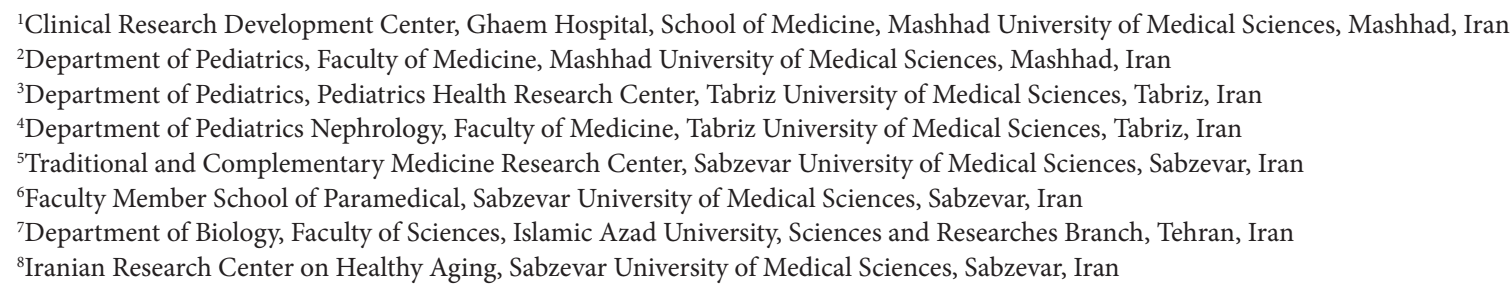

\section{AR T I C L E I N F O}

\section{Article Type:}

Original

\section{Article History:}

Received: 9 December 2016

Accepted: 10 May 2017

Published online: 4 June 2017

\section{Keywords:}

Desmopressin

Oxybutynin

Primary nocturnal enuresis

\begin{abstract}
A B S T RA C T
Introduction: Nocturnal enuresis is known as the most common developmental problems of childhood which has often a familial basis. It is usually benign and gradually disappears with age.

Objectives: This study was conducted on children with primary nocturnal enuresis to compare the efficacy of desmopressin and oxybutynin combination therapy with desmopressin monotherapy.

Patients and Methods: This clinical trial was conducted on 59 patients (age range; 5 to 15 years) with primary nocturnal enuresis who had referred to our center in Tabriz in 2012. The participants were divided into two groups as group 1 (30 participants) and group 2 (29 subjects). For three months, one group received desmopressin and oxybutynin and the other group received desmopressin alone. Descriptive statistics (mean \pm SD and frequency) and chi-square test was conducted.

Results: In the group treated only with desmopressin, $72.4 \%$ of the participants were completely cured in 1 month and $44.8 \%$ in 3 months. In the group treated with combination of desmopressin and oxybutynin, $83.34 \%$ were cured in 1 month and $86.7 \%$ in 3 months. Conclusion: Desmopressin and oxybutynin combination therapy is more effective than desmopressin monotherapy for treating children with enuresis.
\end{abstract}

Implication for health policy/practice/research/medical education:

Desmopressin and oxybutynin combination therapy is more effective than desmopressin monotherapy for treating children with enuresis.

Please cite this paper as: Ravanshad Y, Azarfar A, Ghalegolab-Behbahan A, Mortazavi F, Ahmadzadeh S, Ghorat F, et al. Comparing the efficacy of desmopressin and oxybutynin combination therapy and desmopressin monotherapy in children with primary nocturnal enuresis; a randomized clinical trial. J Renal Inj Prev. 2017;6(4):259-263. DOI: 10.15171/jrip.2017.49.

\section{Introduction}

Enuresis refers to involuntary urination during night sleep which happens in children older than 5 years old. The prevalence of nocturnal enuresis is $15 \%$ to $20 \%$ in 5 -yearold children, which decreases to $1 \%-2 \%$ until the age of 15 years old (1). Nocturnal enuresis is more common in boys than girls (2) and it has two types; (a) Primary nocturnal enuresis which exists since birth; (b) Secondary nocturnal enuresis in which a patient has no nocturnal enuresis at least in the last six months (3). 
In addition, nocturnal enuresis is divided into two groups of mono-symptomatic and non-mono-symptomatic. In mono-symptomatic form, patients have only nocturnal enuresis, but in non-mono-symptomatic, in addition to nocturnal enuresis, patients may have some other symptoms such as frequent urination, dysuria and daytime incontinence (3-5). The etiology of primary nocturnal enuresis is not yet known, but some factors such as stimulatory disorders during sleep, excessive production of urine at night and reduced bladder capacity during sleep are influential factors (5).

Four genes on chromosomes numbers 12, 13, 22 and 8 are identified so far which are related to nocturnal enuresis. The most common way of the disease transmission is autosomal dominant inheritance which has about 90\% penetration. If one of the parents has the experience of nocturnal enuresis, there is a $40 \%$ chance, and if both of them have this experience, a $70 \%$ chance was existed for their child gets enuresis (6).

The influencing factors on the secondary nocturnal enuresis include bladder infection, constipation, diabetes mellitus, diabetes insipidus, intestinal obstruction, neurogenic bladder, seizures and medication side effects. It is important to diagnose primary or secondary types of nocturnal enuresis in patient evaluation because the medication interventions in the secondary type include eliminating the causes (7). The first step in management of patients with nocturnal enuresis is taking history and doing physical examinations. In history taking, patient's urination pattern, including frequency of urination during day and night, daytime incontinence and frequency of nocturnal enuresis in one week, is assessed. The amount of consumed liquid during the day and evening and also before sleeping is assessed as well. The physical examination of patients includes; accurate neurological examination, spinal cord examination for dysraphism and genital examination to check developmental abnormalities of the genitourinary system (3).

Urine analysis to examine probable infection or glucosuria is necessary for all patients with nocturnal enuresis symptoms. It is recommended to take a urinary system sonography test to check urinary system anatomy. To evaluate the bladder function, urine residual measurement after voiding is recommended too (3).

After a confirmed diagnosis of nocturnal enuresis, the disease should be treated. There are three therapeutic methods for nocturnal enuresis (3): (a) Behavioral therapy, (b) treatment with a buzzer alarm, and (c) pharmacological treatment.

Pharmacological therapies which are used for nocturnal enuresis include desmopressin, anticholinergic factors and tricyclic antidepressant drugs $(8,9)$. The efficacy of desmopressin in primary treatment of enuresis is proven in several studies. There are two types of desmopressin; nasal spray and pills. Due to its short onset and fewer side effects, the oral form of desmopressin is more recommended $(8,10)$. The dosage is $20 \mu \mathrm{g}$ every night (for nasal spray) or $0.2 \mathrm{mg}$ (for oral form). Desmopressin intake in $68.8 \%$ of the patients has led to the improvement of nocturnal enuresis
$(11,12)$. It is recommended to take desmopressin for three months, and if no response to treatment is observed, then it can be considered as desmopressin resistant nocturnal enuresis. In $20 \%$ of the patients with nocturnal enuresis, this type of resistance is observed (13-15).

\section{Objectives}

At the moment, there is no specific treatment of choice for primary nocturnal enuresis. As it was expected that the efficacy of combination therapy is higher than monotherapy, this study was conducted to compare the efficacy of combination therapy on nocturnal enuresis. Thus, this study set out to compare the therapeutic effects of desmopressin and oxybutynin combination with desmopressin alone on children with primary nocturnal enuresis.

\section{Patients and Methods}

This clinical trial was carried out on 59 patients (age range: 5 to 15 years old) with primary nocturnal enuresis who had referred to our center in Tabriz in 2012. According to previous studies the sample size was calculated to be 59 people (11). All participants were screened to separate those with primary nocturnal enuresis from the other types of enuresis and were assigned to one of the treatment groups.

The evaluation of children with nocturnal enuresis was done as follows:

(A) Taking a complete history:

1. associated symptoms: primary or secondary, daytime or nocturnal, urinary symptoms.

2. developmental history: the time of acquiring developmental criteria and bladder control.

3. family history: history of having the disease in parents, siblings or having undesirable accidents.

(B) Physical examination:

1. external genitalia examination

2. abdomen examination for existence of kidney or bladder mass

3. neurological examination: peripheral reflexes and perineal area sensation

(C) Laboratory assessments:

1. urine test to measure specific gravity, glucose, blood and protein

2. urine culture

3. blood sugar level

4. serum creatinine level

5. kidney sonography.

After the confirmation of primary nocturnal enuresis diagnosis, the participants were divided into two groups of 29 and 30 people. Group 1 with 30 people received the desmopressin plus oxybutynin combination therapy and group 2 with 29 people received only desmopressin. According to the high price of desmopressin oral pills, desmopressin nasal spray was prescribed with dosage of $10 \mu \mathrm{m}$ in each nostril (before sleeping) for all patients. In addition, oxybutynin was prescribed as oral pills with the dosage of $5 \mathrm{mg}$ (half pill, twice daily). Then during their monthly visits, these patients were monitored by 
nephrologists to check medication side effects, enuresis improvement and reduction in frequency of urination during night.

Medication side effects were assessed by the presence or absence of any clinical complains that had been reported before. Due to the diversity of tolerable side effects of medications, all of the participants were not monitored and analyzed, except those dangerous side effects that led to discontinuation of the treatment in patients.

The final result of the treatment was improvement of nocturnal enuresis which meant having nocturnal bed wetting less than twice per month in a child who had this problem before. The evaluated side effects of desmopressin included seizures (the measurement of sodium level in terms of the risk of hyponatremia), runny nose, nasal congestion and epistaxis, gastrointestinal disturbances such as nausea and stomach cramps and temporary headache. Oxybutynin side effects also included palpitation (ECG checked), dry mouth (xerostomia) and constipation.

The treatment course for each participant was decided to be 3 months. Those participants who did not complete it or refused to come back were excluded from the study. The other exclusion criteria (including confounding factors) were as follows:

1. Neurogenic bladder due to any cause, including tumoral lesions, spinal cord injuries, congenital malformations, sacrococcygeal or urogenital anomalies.

2. Central nerve system dysfunctions including cerebral palsy and mental retardation.

3. Urine concentration defects including renal tubules dysfunction, electrolyte disorders and diabetes.

4. Refusing to voluntarily participate in the study at the beginning or during the study.

Response to treatment and probable side effects were recorded in a questionnaire.

\section{Ethical issues}

The research followed the tenets of the Declaration of Helsinki; informed consent was obtained; and the research was approved by the ethical committee of Tabriz University of Medical Sciences. The research has been also registered in the Iranian registry for clinical trials website (Identifier: IRCT201301117892N4, http://irct.ir).

\section{Statistical analysis}

The collected data were analyzed by SPSS software version 16. Descriptive statistics (mean, standard deviation and frequency) and chi-square test were also applied. The significance level of $P$ was set at 0.05 .

\section{Results}

In this study, 59 patients (age range; 5 to 15 years old) with primary nocturnal enuresis who had referred to our center, were divided into two treatment groups. One group ( $\mathrm{n}=$ 30) was treated with a combination of desmopressin and oxybutynin and the other group $(\mathrm{n}=29)$ was treated with desmopressin alone. The mean age of patients in group one (desmopressin + oxybutynin) was $7.86 \pm 2.41$ years old. The mean age of all participants was $6.89 \pm 2.5$ years old and there was no significant difference between the mean age of the two groups $(P=0.55)$.

In the group treated with desmopressin plus oxybutynin, 17 children (56.7\%) were boys and 13 (43.3\%) were girls and in group treated with desmopressin alone there were 16 (55.2\%) boys and 13 (44.8\%) girls. Therefore, there was no significant difference in gender between the two groups $(P=0.558)$.

In $30.5 \%$ of the participants the father, $25.4 \%$ the mother, $5.1 \%$ the brother and $3.4 \%$ the sister had a family history of nocturnal enuresis. Totally, 35.6\% mentioned that they did not have any family history of this disease. Furthermore, 21 out of 29 participants $(72.4 \%)$ in the group treated with desmopressin alone were completely recovered after 1 month. Three months after treatment in this group, 13 participants $(44.8 \%)$ had completely recovered and in 16 participants (55.2\%) with no improvement were observed. Around, one month after treatment, the result of treatment in the group of desmopressin and oxybutynin was as follows; around 25 out of 30 participants (83.34\%) were completely cured and 5 participants $(16.65 \%)$ were not cured. In treatment evaluation after three months, however, 26 participants $(86.7 \%)$ were completely cured and $13.3 \%$ still suffered from nocturnal enuresis (Figures 1 and 2).

The comparison of results after one month treatment shows a significant difference between the proportions of cured people in the two groups. The proportion of cured subjects in combination therapy group was more than those in monotherapy group $(P=0.047)$. The result of evaluation after three months showed, combination therapy was also more effective than monotherapy $(P<0.001)$.

In combination therapy group, no significant association between gender and the result of treatment after one month was detected, while the treatment response rate in this group was $84 \%$ in girls and $82.3 \%$ in boys $(P=0.88)$. However, there was a significant association between age and treatment results. In this group, the treatment result after one month was better in younger children. The maximum recovery rate was in participants with age of seven years old and younger.

In combination therapy group, there was no significant

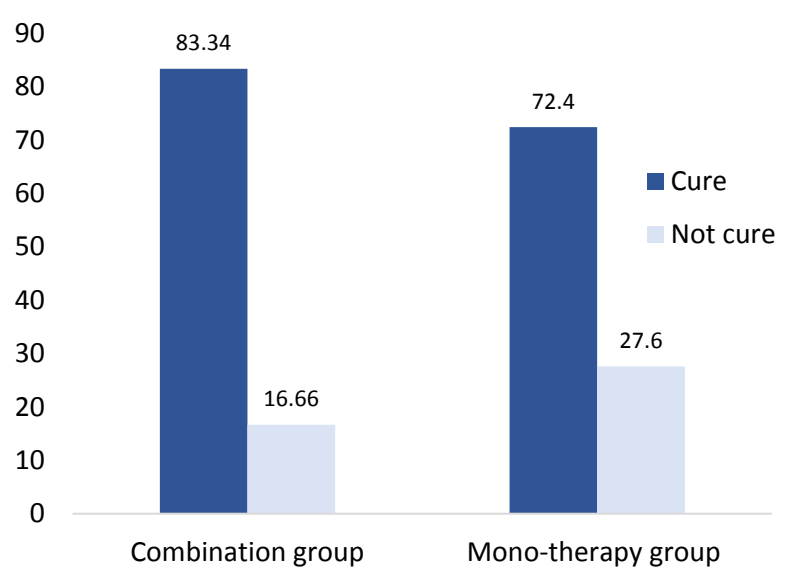

Figure 1. Comparison of the treatment result after one month in combination and mono-therapy groups. 


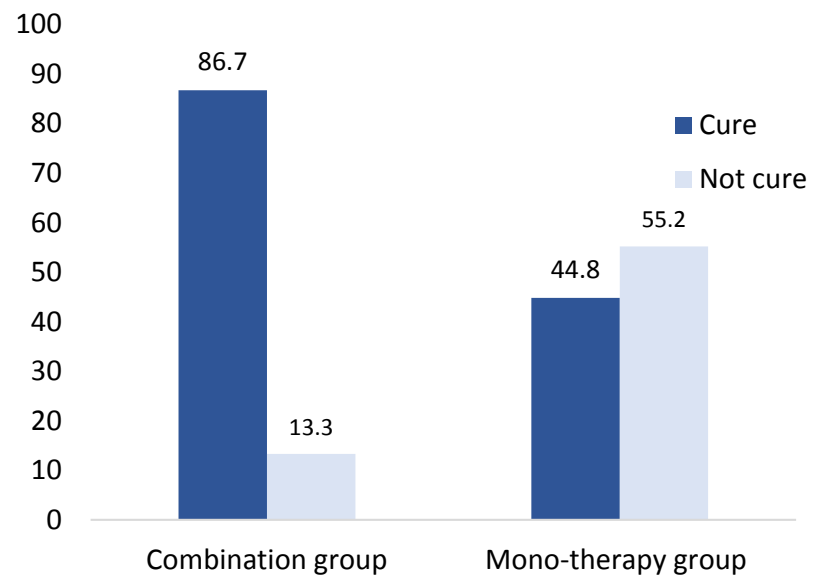

Figure 2. Comparison of treatment results after three months in combination and mono-therapy groups.

association between age, sex and the treatment results after three months $(P>0.05)$. Additionally, in monotherapy group no significant association between age, sex and treatment result after one and three months was detected $(P>0.05)$.

\section{Discussion}

Evaluating treatment in desmopressin group showed that $72.4 \%$ of the participants were completely recovered after 1 month and $44.8 \%$ after 3 months. In desmopressin and oxybutynin combination group $83.34 \%$ were completely cured after 1 month and $86.7 \%$ after 3 months. The results of Austin colleagues' study on comparison of the efficacy of treatment with desmopressin and tolterodine (an anticholinergic) combination and treatment with desmopressin alone, demonstrated that in combination therapy group, bed wetting was $66 \%$ less than the other group (16). This finding is consistent with the result of our study, and it indicates that treatment of nocturnal enuresis with a combination of desmopressin and an anticholinergic is better than treatment with desmopressin alone.

The results of a study conducted by Radvanska et al, on the efficacy of combination therapy with oxybutynin and desmopressin in patients with enuresis who had no treatment response to desmopressin alone, showed that two drugs combination therapy can reduce the frequency of nocturnal bed wetting in desmopressin resistant patients by $43 \%$ (17). In the study, the patients had administered desmopressin before they took combination therapy with desmopressin and oxybutynin (17). However, in our study, patients were assigned randomly to one of the treatment groups. We believe this is the reason that the response to treatment rate in these two studies is different.

Martin and colleagues compared the efficacy of desmopressin and oxybutynin combination therapy with oxybutynin monotherapy. They found out a significant difference between mean bladder adaptation and urine flow pressure in patients treated with oxybutynin alone. They concluded that the efficacy of combination therapy with desmopressin plus oxybutynin was better than oxybutynin alone $(16,18)$, which is consistent with the results of our study. However, we did not study the mean bladder adaptation and urine flow pressure in the studied patients. In addition, in a study by Lee and colleagues, the combination therapy with desmopressin plus oxybutynin had a remarkable effect on speed and effectiveness of the treatment on children with nocturnal enuresis $(19,20)$. Results of the present study show that in desmopressin group, $72.41 \%$ were cured after 1 month and $44.8 \%$ after 3 months. Falak-ul-aflaki and colleagues studied the efficacy of treatment with desmopressin in 5-14 years old children with nocturnal enuresis and observed that $82.5 \%$ of the patients were completely cured (21). The treatment rate after 1 month in our study is not consistent with Falak-ulaflaki and colleagues' work, but treatment percentage after 3 months is consistent.

Additionally, no significant difference age, sex and efficacy of the treatment with desmopressin therapy alone was detected in our study. However, in treatment evaluation after 1 month of combination therapy group, the treatment response in children 7 years old and younger was higher. This may be due to the difference between the proportion of children younger and older than 7 years old in this study. However, more studies with larger sample sizes is necessary to confirm this hypothesis. Falak-ul-aflaki and colleagues found no significant difference between age, sex, stress level and medication efficacy (21).

Although the side effects such as dry mouth and constipation did not hinder the treatment, 20 participants in combination therapy group reported drug side effects. In monotherapy group side effects were observed in 23 participants. There was no significant difference between type of treatment and side effects $(P=0.213)$, which is consistent with Lee and colleagues' study (19).

\section{Conclusion}

In children with nocturnal enuresis, desmopressin plus oxybutynin combination therapy is more effective than desmopressin monotherapy. Therefore, the combination therapy should be prioritized. Our suggestions for future work on this subject are as follows;

- Conducting a similar study with a larger sample size so that the results can be generalized with more certainty.

- Conducting a similar study to evaluate the treatment results with other drugs and compare the results with Desmopressin treatment.

- Conducting a similar study to assess the reduction in frequency of nocturnal enuresis and the amount of improvement in two treatment groups (desmopressin plus oxybutynin and desmopressin alone).

- Conducting a similar study and follow up the patients after cessation of drug treatment.

- Conducting a similar study and assessment of the frequency of side effects according to the type of side effects in each treatment group.

Limitations of the study

A limitation of our study was small proportion of patients. 
We suggest multi-centric studies on this aspect of kidney disease.

\section{Acknowledgements}

This article was derived from M.D thesis by Sonia Ahmadzadeh (\#656842). The authors express their gratitude to all colleagues and nurses who assisted them in this work. The authors thank Seyed Muhammed Hussein Mousavinasab for his sincere participation in editing the text.

\section{Ethical considerations}

Ethical issues (including plagiarism, data fabrication, double publication) have been completely observed by the authors.

\section{Conflicts of interest}

The authors declared no competing interests.

\section{Authors' contribution}

YR and AA contributed to the arrangement of the study, conducted the data collecting, and served as the lead author of the manuscript. MV, AG, FM and SA wrote some parts of the draft. FG, SF and EI participated in the arrangement of the study, conducted the statistical analysis, and finalized the manuscript. All authors read and approved the final manuscript.

\section{Funding/Support}

This study was supported by a research grant from Tabriz University of Medical Sciences (Grant \#656842).

\section{References}

1. Azarfar A, Esmaeili M, Naseri M, Ghane F, Ravanshad Y, Vejdani M, et al. Comparison of combined treatment with desmopressin plus oxybutynin and desmopressin plus tolterodine in treatment of children with primary nocturnal enuresis. J Renal Inj Prev. 2015;4:80-6. doi: 10.12861/ jrip.2015.16.

2. Byrd RS, Weitzman M, Lanphear NE, Auinger P. Bedwetting in US children: epidemiology and related behavior problems. Pediatrics. 1996;98:414-9.

3. Caione P, Arena F, Biraghi M, Cigna RM, Chendi D, Chiozza ML, et al. Nocturnal enuresis and daytime wetting: a multicentric trial with oxybutynin and desmopressin. Eur Urol. 1997;31:459-63.

4. Naseri M, Hiradfar M. Monosymptomatic and nonmonosymptomatic nocturnal enuresis: a clinical evaluation. Arch Iran Med. 2012;15:702. doi: 0121511/AIM.0011

5. Yeung CK, Chiu HN, Sit FK. Bladder dysfunction in children with refractory monosymptomatic primary nocturnal enuresis. J Urol. 1999;162:1049-54.
6. Geary DF, Schaefer F. Comperhensive Pediatric Nephrology Philadelphia: Elsevier; 2008

7. Robson WL, Leung AK, Van Howe R. Primary and secondary nocturnal enuresis: similarities in presentation. Pediatrics. 2005;115:956-9.

8. Neveus T. Oxybutynin, desmopressin and enuresis. J Urol. 2001;166:2459-62.

9. Lundmark E, Nevéus T. Reboxetine in therapy-resistant enuresis: a retrospective evaluation. Scand J Urol Nephrol. 2009;43:365-8. doi: 10.3109/00365590903099959.

10. Ghasemi K, Esteghamati M, Mohammadzadeh M, Zare S. Desmopressin versus oxybutynin for nocturnal enuresis in children in Bandar Abbas: a randomized clinical trial. Electron Physician. 2016;8:2187. doi: 10.19082/2187

11. Harris AS. Clinical experience with desmopressin: efficacy and safety in central diabetes insipidus and other conditions. J Pediatr. 1989;114:711-8.

12. Kim S-O, Yu H, Kwon D. Efficacy of desmopressin to treat nocturnal polyuria in elderly men: effects on sleep quality. Urol Int. 2016;96:438-42. doi: 10.1159/000443968

13. Terho P. Desmopressin in nocturnal enuresis. J Urol. 1991;145:818-20

14. Nevéus T. Nocturnal enuresis-theoretic background and practical guidelines. Pediatric nephrology. 2011;26:120714. doi: 10.1007/s00467-011-1762-8.

15. Walle JV, Rittig S, Bauer S, Eggert P, Marschall-Kehrel D, Tekgul S. Practical consensus guidelines for the management of enuresis. Eur J Pediatr. 2012;171:971-83. doi: 10.1007/s00431-012-1687-7.

16. Austin PF, Ferguson G, Yan Y, Campigotto MJ, Royer ME, Coplen DE. Combination therapy with desmopressin and an anticholinergic medication for nonresponders to desmopressin for monosymptomatic nocturnal enuresis: a randomized, double-blind, placebo-controlled trial. Pediatrics. 2008;122:1027-32. doi: 10.1542/peds.2007-3691.

17. Radvanska E, Kovacs L, Rittig S. The role of bladder capacity in antidiuretic and anticholinergic treatment for nocturnal enuresis. J Urol. 2006;176:764-8.

18. Martin-Crespo R, Luque R. In which patients is the oxybutynin-desmopressin combination therapy indicated? Cir Pediatr. 2003;16:181-5.

19. Lee T, Suh H. Comparison of effect of treatment of primary nocturnal enuresis with oxybutynin plus desmopressin or desmopressin alone or imipramine a lone: a randomized controlled clinical trial. J Urol. 2005;174:1084-7.

20. Kwak KW, Lee Y-S, Park KH, Baek M. Efficacy of desmopressin and enuresis alarm as first and second line treatment for primary monosymptomatic nocturnal enuresis: prospective randomized crossover study. J Urol. 2010;184:2521-6. doi: 10.1016/j.juro.2010.08.041

21. Falak-ul-Aflaki B, Sharifi F, Torabi Z, Moezi F. Evaluation of Theraputic effect of desmopressin on enuresis in children. Journal of Zanjan University of Medical Science \& Health Services. 2007;15:11-6. [Persian].

Copyright (c) 2017 The Author(s); Published by Nickan Research Institute. This is an open-access article distributed under the terms of the Creative Commons Attribution License (http://creativecommons.org/licenses/by/4.0), which permits unrestricted use, distribution, and reproduction in any medium, provided the original work is properly cited. 